\title{
O impacto da psoríase na qualidade de vida dos portadores: estigmatização e prejuízos biopsicossociais
}

\author{
The impact of psoriasis on the quality of life of patients: stigmatization and \\ biopsychosocial damage
}

\section{El impacto de la psoriasis en la calidad de vida de los pacientes: estigmatización y daño biopsicosocial}

Alessandra Ornella Paraiso1*, Gabriela Luiza Spohr², Amanda Mendonça Reginaldo1, Ana Luiza Oliveira do Carmo $^{3}$, Caio Siqueira Honorato ${ }^{4}$, Thays Nascimento Ferreira ${ }^{5}$, Lara Nunes Belo ${ }^{6}$, Mateus Alves Fernandes Lopes ${ }^{7}$, Ana Cláudia Soares de Souza Meira ${ }^{8}$, Milena Ferreira Gandra ${ }^{7}$.

\section{RESUMO}

Objetivo: Fazer uma análise de estudos abordados cientificamente em relação à psoríase, uma doença crônica da pele, sem cura, que resulta em áreas escamosas, muitas vezes com coceira nas manchas. Revisão bibliográfica: Esse artigo mostrou que além da psoríase agir de forma danosa na qualidade de vida do indivíduo acometido diante aos problemas físicos gerados por essa afecção dermatológica, ela ainda interfere na saúde mental do paciente, uma vez em que esta patologia atinge a pele, uma das partes mais expostas e visíveis do corpo afetando as relações pessoais e a forma como o indivíduo é visto perante a sociedade que por falta de conhecimento muitas vezes temem que os doentes possam Ihe inf ectar causando assim um afastamento social do paciente e porconsequências danos psíquicos no indivíduo. Considerações finais: Nota-se que pela psoríase ser uma doença que causa danos em diversas áreas do indivíduo, é importante que o paciente seja acolhido por uma equipe multiprofissional e possua uma rede de apoio para assim enfrentar o tratamento da melhor forma possível, diminuindo possíveis impactos psicológicos e trazendo maior qualidade de vida.

Palavras-chave Psoríase, Qualidade de vida, Doença.

\section{ABSTRACT}

Objective: Analyze studies scientifically addressed in relation to psoriasis, a chronic, incurable skin disease that results in scaly areas, often with itchy patches. Bibliographic review: This article showed that in addition to psoriasis acting in a harmful way on the quality of life of the individual affected by the physical problems generated by this dermatological condition, it also interferes with the patient's mental health, since this pathology affects the skin, one of the most exposed and visible parts of the body, affecting personal relationships and the way the individual is seen in society that, due to lack of knowledge, of ten fear that patients can infect them, thus causing a social withdrawal from the patient and psychological damage consequences in the individual. Final considerations: It is noted that because psoriasis is a disease that causes damage in several areas of the individual, it is important that the patient is welcomed by a multidisciplinary team and has

\footnotetext{
1 Pontifícia Universidade Católica de Minas Gerais (PUC-MG), Betim - MG.

${ }^{*}$ E-mail: alessandra.ornella@hotmail.com

2 Universidade Católica de Pernambuco (UNICAP), Recife - PE.

3 Faculdade Dinâmica do Vale do Piranga (FADIP), Ponte Nova - MG.

${ }^{4}$ Centro Universitário de Valença (UNIFAA), Valença - RJ.

5 Universidade Nilton Lins (UNL), Manaus - AM.

6 Universidade de Itaúna (UIT), Itaúna - MG.

7 Centro Universitário de Caratinga (UNEC), Caratinga - MG.

8 Universidade do Estado de Minas Gerais (UEMG), Passos - MG.
}

SUBMETIDO EM: 9/2021 | ACEITO EM: 10/2021 | PUBLICADO EM: 11/2021


a support network to face the treatment in the best possible way, reducing possible psychological impacts and bringing a better quality of life.

Key words: Psoriasis, Quality of life, Disease.

\section{RESUMEN}

Objetivo: Analizar estudios científicamente abordados en relación con la psoriasis, una enfermedad cutánea crónica e incurable que produce áreas escamosas, a menudo con parches que pican. Revisión bibiliográfica: Este artículo mostró que además de que la psoriasis actúa de forma nociva sobre la calidad de vida del af ectado ante los problemas físicos que genera esta condición dermatológica, también interfiere con la salud mental del paciente, ya que esta patología afecta la piel, una de las partes más expuestas y visibles del cuerpo, af ectando las relaciones personales y la forma en que se ve al individuo en la sociedad que, por desconocimiento, muchas veces temen que los pacientes puedan contagiarlos, provocando así un retraimiento social del consecuencias del daño psicológico y del paciente en el individuo. Consideraciones finales: Se observa que debido a que la psoriasis es una enfermedad que causa daño en varias áreas del individuo, es importante que el paciente sea recibido por un equipo multidisciplinar y cuente con una red de apoyo para enfrentar el tratamiento de la mejor manera posible, reduciendo posibles impactos psicológicos y trayendo una mejor calidad de vida.

Palabras clave: Psoriasis, Calidad de vida, Enfermedad.

\section{INTRODUÇÃO}

A psoríase é uma dermatose inflamatória crônica da pele, não contagiosa e de origem desconhecida. No entanto, acredita-se que ela possa estar relacionada ao sistema imune, às interações com o ambiente e à herança genética. A principal manifestação clínica da doença é a intensa e rápida proliferação de células da epiderme, com a formação de lesões eritêmato-descamativas, com diversas formas de apresentação: vulgar ou em placas, ungueal, palmoplantar, invertida, artropática, pustulosa, gutata e eritrodérmica, sendo a forma em placas a mais prevalente, configurando 90\% dos casos (SILVEIRA MEB, et al., 2017). Dessa forma, as lesões visíveis em diversas regiões do corpo podem limitar as atividades cotidianas desses indivíduos por conta da vergonha, da insegurança e da frustração com o próprio corpo (ŠMEJKALOVÁ J, et al., 2020, GARCÍA-SÁNCHEZ L, et al., 2017).

A doença af eta ambos os sexos igualmente e abrange todas as faixas etárias, tod avia, estudos apontaram 33 anos como sendo a idade média de início, sendo que $75 \%$ dos casos ocorreram antes dos 46 anos de idade. Outros ensaios constataram um início bimodal, com dois picos da doença ao longo da vida, um mais cedo, entre os 16 e 22 anos e outro mais tarde, entre 57 e 60 anos de idade (ORGANIZAÇÃO MUNDIAL DA SAÚDE (OMS), 2016).

As implicações na vida do paciente com psoríase vão além das consequências biológicas, af etando as vivências emocionais, sociais e pessoais desses indivíduos e sendo a origem de sentimentos ruins como 0 medo e a frustração. Desse modo, a doença exige uma abordagem biopsicossocial e multiprofissional para que haja a readaptação e a reorganização das atividades diárias e seja garantida a integridade da qualidade de vida (GUERREIRO TN, et al., 2018; SILVA B e FARO A, 2019).

A originalidade da patologia ainda não é totalmente compreendida, pois apresenta-se em períodos de exacerbação e remissão dos sintomas, tendo sua causa pouco conhecida. A psoríase apresenta predisposição genética, não existindo um desencadeante isolado e dependendo de um estímulo exógeno para a manifestação. Além disso, o tratamento da psoríase depende da clínica, gravidade e extensão da doença, e inclui medicamentos tópicos, fototerapia e medicamentos sistêmicos (tradicionais e imunobiológicos). Assim sendo, pacientes acometidos pela patologia apresentam grande impacto na qualidade de vida, repercutindo nas atividades diárias, limitando-as ou mesmo impedindo-as. O impacto 
psicológico e emocional causado pela psoríase, nem sempre está relacionado à extensão/gravidade da doença (GUERREIRO TN, 2018; SCACCABAROZZI L, 2016).

Diante do exposto, o objetivo do presente trabalho visou realizar uma revisão bibliográfica da literatura acerca das manifestações dermatológicas da psoríase e como ela impacta a qualidade de vida dos pacientes .

\section{REVISÃO BIBLIOGRÁFICA}

\section{Fisiopatologia}

A psoríase é uma doença dermatológica autoimune não contagiosa, que cursa com uma apresentação sistêmica inflamatória e crônica recorrente da pele e articulações, no qual, pode acometer órgãos adjacentes. Ademais, possui um caráter cíclico, isto é, os sintomas desaparecem e reaparecem periodicamente. Neste contexto, histologicamente, a psoríase apresenta uma hiperproliferação celular (JESUS AN, et al., 2016).

Atualmente, a etiologia concreta da psoríase ainda é desconhecida, porém, sabe-se que a doença apresenta um curso multif atorial, na qual, aborda uma correlação ao sistema imunológico e às interações com o meio ambiente e à suscetibilidade genética. Ademais, estudos mostram que, a incidência do aparecimento das lesões dermatológicas, podem estar relacionados quando os linfócitos Tliberam substâncias inflamatórias e formadoras de vasos. Isto pois, essas respostas imunológicas podem acarretar uma dilatação dos vasos sanguíneos da pele e consequentemente uma infiltração da pele com neutrófilos. Dessa forma, como as células da pele acabam sendo acometidas, seguidamente ocorre uma rapidez no ciclo evolutivo, no qual, gera uma elevada produção de escamas em consequência à imaturidade das células (MACHADO ER, et al., 2019)

Pode-se dizer que, existem diversos fatores extrínsecos, nos quais, podem desencadear o surgimento ou a piora das lesões, dentre eles, o tempo frio, as infecções e o estresse. Além disso, há uma série de particularidades que também podem estar relacionadas com a gravidade da doença, como por exemplo, 0 alcoolismo, depressão, obesidade, diabetes mellitus, hipertensão arterial, síndrome plurimetabólica, colite e artrite reumatoide. Face a isto, a qualidade de vida dos pacientes portadores de psoríase, sofrem grandes impactos quanto aos aspectos psicossociais, atividades do dia a dia e posteriormente nas relações sociais e interpessoais (MOSCARDI RE, et al., 2017).

As formas de apresentação clínica da psoríase podem se dividir em diversas formas, logo cita-se as principais como a psoríase vulgar, sendo a manifestação mais comum da doença, na qual, apresenta diversas formas de placas secas eritematosas e descamação esbranquiçada; psoríase ungueal, nestes casos o paciente é afetado diretamente nas unhas das mãos e dos pés; psoríase do couro cabeludo, no qual, apresenta áreas avermelhadas com escamas espessas branco-prateadas, principalmente após coçar o couro cabeludo; a psoríase gutata normalmente é desencadeada por infecções bacterianas, como exemplo as amigdalites.Por fim, a psoríase artropática, que além da inflamação na pele e da descamação, a artrite psoriática, leva a um quadro intenso com fortes dores nas articulaçõ es (MENDES RCA, 2019)

É válido ressaltar que, estudos médicos avançados mostram que, mesmo a psoríase não apresentando um curso curativo, há expectativas diante de novos tratamentos, que abrangem como função a redução da gravidade das lesões, logo tendem a levar a patologia dermatológica a desaparecer quase, ou totalmente. Dessa maneira, em especial no Brasil, um novo fármaco foi recém-aprovado, conhecido como ixequizumabe, no qual, propõe-se devolver a qualidade de vida ao paciente. Porém, é um medicamento de alto custo, pois é uma injeção aplicada mensalmente pela própria pessoa na barriga ou na perna, tendo indicação para as formas mais graves e pacientes que já falharam ao tratamento convencional (COMISSÃO NACIONAL DE INCORPORAÇÃO DE TECNOLOGIAS NO SUS (CONITEC, 2020).

Dessa forma, atualmente existem mais de 50 métodos que avaliam a gravidade da psoríase, entretanto, diversos instrumentos de avaliação ainda não foram validados adequadamente, tampouco apresentam uniformidade entre si. Por isso, os principais mecanismos de observação de gravidade da patologia citada são, em primeiro lugar o Psoriasis Area and Severity Index (PASI), no qual, é o instrumento mais utilizado, pois as placas dermatológicas são analisadas segundo critérios como infiltração e descamação. Logo, a 
gravidade da doença é dada para cada item de acordo com uma escala de intensidade que varia de 0 a 4 . Por conseguinte, tem-se o Body Surface Area (BSA), que é usado para a avaliação de acordo com o número de áreas lesionadas, como exemplo palma das mãos. Já o Physician's Global Assessment (PGA), é uma escala com números ordinais, em que se identificam integralmente uma psoríase sem lesão e até um quadro muito grave. Neste caso os critérios considerados se englobam no eritema, infiltração e descamação (SOCIEDADE BRASILEIRA DE SOCIEDADE BRASILEIRA DE DERMATOLOGIA (SBD), 2020).

Ademais, o Dermatology Life Quality Index (DLQI), também conhecido como índice de qualidade de vida em dermatologia, é um questionário que aborda como objetivo a realização da medição do problema atual, isto é, avaliar o impacto na qualidade de vida frente a doença. Além dis so, o índice contém 10 questões que estão relacionadas às experiências vivenciadas pelos indivíduos portadores de psoríase, na semana precedente à aplicação do questionário (SBD, 2020).

\section{Manifestações clínicas associadas}

A placa de ateroma e a progressão das doenças inflamatórias crônicas possuem semelhanças nos seus fatores imunológicos responsáveis pela formação, como na psoríase e, com isso, permitiu estabelecer uma relação com a incidência de doenças cardiovasculares. Com este achado, pacientes portadores de psoríase grave possuem alta frequência em hipertensão, diabetes, doença cardiovascular, artrite psoriásica e risco aumentado para infarto agudo do miocárdio (LIMA EA e LIMA MA, 2011).

Ademais, foi identificado associação entre a doença e hipertensão, diabetes, obesidade, dislipidemia e infarto agudo do miocárdio, especialmente em homens de 35 e 50 anos de idade a partir do estudo com 340 pacientes com psoríase e 6.643 controles. Uma avaliação de 16.851 pacientes com psoríase constatou aumento na concentração de triglicerídeos e colesterol total, associado a diminuição da concentração sérica de HDL, quando comparados com controles (COHEN AD, et al., 2006).

Acrescentando a estes itens, apontaram a existência da relação entre sintomas depressivos e a percepção de gravidade da doença, o que reforça os achados da literatura em direção às consequências e incapacidades geradas pela psoríase. Além disso, assim como demonstram outras evidências, observou-se que o maior uso da estratégia de supressão emocional esteve relacionado à sintomatologia depressiva (SANTOS VP, et al., 2016; SILVA B e FARO A, 2019).

Portanto, visto que as evidências quanto à presença de sintomas depressivos ressaltam o alto índice de comprometimento psicoemocional desencadeado pela doença de pele é importante destacar a necessidade de acompanhamento psicológico desses pacientes (SILVA B e FARO A, 2019).

\section{Impactos na saúde mental}

A pele é parte essencial da imagem corporal e é também um instrumento de manifestação cultural, então o comprometimento deste órgão por lesões, principalmente, se forem visíveis pode acarretar sofrimento psíquico (CALVETTI PU, et al., 2020).

Nessa perspectiva, as manifestações dermatológicas da psoríase podem estar relacionadas com o aumento do estresse, da depressão e da ansiedade nesse grupo. E esse ciclo doença-impacto na saúde mental acaba por ser intensificado, pois é comprovado que o estresse está diretamente associado com a piora dos sintomas dapsoríase (CALVETTI PU, et al., 2017). Além disso, o acúmulo de sentimentos negativos em relação a si mesmo tende a fragilizar ainda mais o emocional desses indivíduos, os tornando mais suscetíveis a problemas como a depressão (SILVA B, et al., 2019; RUA MO, 2021).

Além disso, a incidência de problemas psicológicos advindos da psoríase independe do grau da mesma, pois os fatores de piora na saúde mental estão mais relacionados com a interação social e a ansiedade advinda da mesma. Sendo que as pessoas af etadas por essa condição possuem uma maior propensão ao uso e abuso de drogas, como o álcool e o tabaco (BULAT V, et al., 2020; PICO CM, 2016).

Assim, fica evidente o impacto mental ocasionado por essa patologia e os ef eitos da mesma na qualidade de vida dessas pessoas, evidenciando, desse modo, a necessidade dessas pessoas de serem assistidas por 
uma equipe multiprofissional desde o descobrimento da doença (KWAN Z, et al., 2017; ARMSRTONG AW e READ C, 2020).

\section{Observações a longo prazo}

Ao definir qualidade de vida deve-se considerar diversos aspectos, a fim de obter um conceito amplo e assertivo. Posto isto, é necessário conciliar positivamente as diferentes esferas: físico, mental, espiritual, social, cultural e ambiental para que se alcance o sentir-se bem proveniente de uma boa qualidade de vida (CALVETTI PU, et al., 2017).

Quanto aos efeitos tardios da doença, pacientes com psoríase de longa data tendem a sofrer com problemas de estigma. Sendo assim, a psoríase deve ser abordada como uma patologia de consequências múltiplas e que atinge diferentes contextos. Uma vez que sua estigmatização interfere no convívio social do indivíduo e sua autoimagem, isto é, o conceito que o indivíduo tem de si mesmo descrito pelo filósofo e psicólogo William James como "self', em Theory of the self de 1992 (CALVETTI PU, et al., 2017).

De acordo com o Ministério da Saúde, há um impacto relevante na qualidade de vida dos pacientes acometidos pela psoríase. Os danos físicos e mentais dessa doença são semelhantes a outras patologias crônicas importantes, a exemplo do câncer, da artrite e da depressão. Por conseguinte, observa-se com maior frequência alterações psiquiátricas nesses pacientes e, portanto, estão mais susceptíveis a diferentes níveis de ansiedade com atitudes de esquiva, fuga e perda do autocontrole. Não obstante, há também o risco de comorbidades importantes, nos casos mais graves, como depressão severa e ideações suicidas. Isso, ao se comparar os efeitos de longo prazo da psoríase às demais dermatoses crônicas e suas complicações (MINISTÉRIO DA SAÚDE, 2021).

O Ministério da Saúde, ainda afirma que mesmo em áreas pequenas quando acometidas, a exemplo das regiões palmar e plantar, podem gerar prejuízo na qualidade de vida dos indivíduos acometidos pela psoríase. Ademais, 71\% desses pacientes têm sua vida sexual af etada devido ao próprio juízo de valor negativo e aos estigmas sociais delegados à doença. Faz-se, portanto, imprescindível o tratamento e acompanhamento psiquiátrico para prevenir os sintomas e comorbidades provenientes da depressão. Desse modo, a psicoterapia é uma alternativa terapêutica importante no controle da doença e suas repercussões (MINISTÉRIO DA SAÚDE, 2021).

Se torna, então, de suma importância a identificação dos estágios iniciais da doença para que o encaminhamento adequado ao profissional de saúde e atendimento especializado seja feito na Atenção Básica a fim de obter o melhor resultado de medidas paliativas e terapêuticas evitando, assim, o mau prognóstico. Algumas medidas podem ser adotadas a fim de minimizar os impactos negativos proveni entes da psoríase, tais como: garantir tratamento de doenças cutâneas no sistema de saúde universal; promover campanhas de conscientização popular acerca dos cuidados necessários referentes a patologia e campanhas contra a estigmatização social associada a psoríase; investir em pesquisas que busquem melhor esclarecer a etiologia da doença; desenvolver tratamentos e medidas paliativos contra a psoríase na finalidade de aumentar a qualidade de vida dos indivíduos acometidos (OMS, 2016; CANHA RIO, 2019).

\section{Estigmas}

O impacto físico na pele é visível em portadores de psoríase. Essa diferença é vista por muitos como algo indesejável e repugnante, além do medo da doença ser contagiosa, criando um estigma. Como resultado, esses portadores sof rem preconceito social, sendo discriminados de diversas formas. Por exemplo, há relatos de pessoas com receio de se expor e ser impedidas de ir à piscina. Isso mostra que o indivíduo se sente rejeitado e envergonhado pelo outro, o que af eta a sua qualidade de vida, pois o medo do julgamento os leva a abrir mão de práticas prazerosas (GUERREIRO TN, 2018, MELO MS, et al., 2019).

Por mais que a doença não seja contagiosa, é frequente a repulsa no meio social. Sentimentos como baixa estima, inconformismo e desprezo são pertinentes em indivíduos diagnosticados com psoríase devido ao preconceito, desencadeando o isolamento social. O fato de se expore estar sob os olhares de outras pessoas, sejam eles curiosos ou julgadores, os deixam incomodados. Sendo assim, esse estigma diante as lesõ es 
podem comprometer o psicológico de uma pessoa acometida por transtorno de pele, podendo ter impactos ainda maiores como, por exemplo, o aparecimento de distúrbios emocionais como depressão e ansiedade (GUERREIRO TN, 2018; BULAT V, 2020; SILVA B e FARO A, 2019).

Além disso, a psoríase tem relação direta com os aspectos emocionais. O estresse contribui para o surgimento ou agravamento dessa dermatose. Isso mostra que os estigmas favorecem o aparecimento de lesões, pois em eventos constrangedores geram estresse e a forma como o doente lida é refletido na pele. Como evidenciam os estudos desenvolvidos na população, $56,5 \%$ associaram o estresse ao início da dermatose e $67,2 \%$ manifestaram estresse no último ano. Dessa forma, é perceptível que além da intervenção medicamentosa, é necessário associar um tratamento psicoterápico (CALVETTI PU, 2017; SILVEIRA MEB, 2017)

Logo, é notório como a estigmatização compromete a qualidade de vida de sujeitos com psoríase. Esta enf ermidade pode comprometer o psicológico, o fís ico e a rotina desses doentes, sendo fonte de sentimentos negativos como culpa e medo. Nessa perspectiva, muitos deixam o convívio social para evitar situações constrangedoras, o que limita a sua vivência seja no trabalho ou no clube. Dessa forma, é impres cindível a readaptação social para o enfrentamento desse estigma no cotidiano (GUERREIRO TN, 2018).

\section{CONSIDERAÇÕES FINAIS}

A psoríase é uma patologia dermatológica crônica relativamente comum que promove um impacto negativo sobre a qualidade de vida dos pacientes, influenciando em aspectos diversos, como físico, emocional, social e pessoal. Portanto, diante desta doença há a necessidade de que o paciente seja acolhido por uma equipe multidisciplinar que promova intervenções farmacológicas bem como intervenções biopsicossociais para que assim o paciente seja assistido na sua totalidade e tenha uma melhor adesão ao seu tratamento, pois apesar de ainda não existir a cura para a psoríase, as opções terapêuticas disponíveis são capazes de controlar a doença, reduzindo os danos nessas pacientes.

\section{REFERÊNCIAS}

1. ARMSTRONG AW, READ C. Pathophysiology, clinical preservation and treatment of psoriasis. American Medical Association (AMA). Jama, 2020; 323(19): 1945.

2. BULATV. Study on the impact of psoriasis on quality of life: psychological, social and financialimplications. Psychiatria Danubina, 2020;32(4): 155.

3. CALVETTI PU, et al. Biopsychosocial aspects and quality of life of people with chronic dermatoses. Psicologia, Saúde \& Doença, 2017; 18(2):297.

4. CANHARIC. Intervenção farmacêutica em doenças autoimunes: psoríase e lúpus. Universidade do Algarve, 2019.

5. COMISSÃO NACIONAL DE INCORPORAÇÃO DE TECNOLOGIAS NO SUS (CONITEC). Protocolo Clínico e $\begin{array}{lllll}\text { Diretrizes Terapêuticas da } & \text { Psoríase. } & \text { Disponível }\end{array}$ http://conitec.gov.br/images/Protocolos/Publicacoes_MS/PCDT_Psorase_Final_ISBN_21-08-2020.pdf. Acesso em:4 de março de 2021

6. GARCÍA-SÁNCHEZ L, et al. Calidad de vida en el paciente con psoriasis. Gac. Med. Mex.,2017; 153(2):185-189

7. GUERREIRO TN, et al. Alterações no cotidiano de pessoas acometidas por psoríase. Revista Enfermagem UERJ, 2018;26: 28332.

8. JESUS AN, et al. Impacto da psoríase na qualidade de vida dos pacientes em tratamento. Rev. In., 2016.

9. KWAN Z, et al. Determinants of quality of life and psychological status in adults with psoriasis. Archives Of Dermatological Research. Springer Science and Business Media LLC , 2018; 310(5):443

10. LIMA EA, LIMA MA. Imunopatogênese da psoríase: revisando conceitos. An. Bras. Dermatol., 2011; 86(6):1151-8.

11. MACHADO ER, et al. Psoríase: uma revisão sistemática da literatura. Rev Inic Cient e Ext., 2019;2(Esp.1).

12. MELO MS, et al. Influência de fatores emocionais nas doenças crônicas de pele: $O$ estresse como gatilho para o desenvolvimento, reincidência ou agravamento da psoríase. Rev. Mult. Psic.,2019; 13(46):584-608.

13. MENDES RCA. Processos de Regulação Emocional em Pessoas com o Diagnóstico de Psoríase. Instituto Superior Miguel Torga, 2019.

14. MINISTÉRIO DA SAÚDE. Protocolo clínico e diretrizes terapêuticas da Psoríase. Brasília, 2021.

15. MOSCARDI RE, et al. Psoríase: etiologia, diagnóstico e tratamento. Rev. Unin., 2017.

16. ORGANIZAÇÃO MUNDIAL DA SAÚDE (OMS). Relatório Global sobre a psoríase. Brasil: Psoríase Brasil. 2016. Disponível em: https://apps.who.int/iris/bitstream/handle/10665/204417/9789241565189por.pdf?sequence=17\&isAllowed=y. Acessado em:23 de Maio de 2021. 
17. PICO CM. O Impacto da psoríase no autoconceito e na socialização da pessoa: Uma Revisão Integrativa Da literatura. Revista Investigação em Enfermagem, 2016;2(17):39-46

18. RODRIGUES JM, et al. Estresse e psoríase:novas abordagens no tratamento. Revista Eletrônica Acervo Científico, 2020;12: 4638 .

19. RUA MO, et al. Influências da depressão na psoríase: uma relação bidirecional. Revista Eletrônica Acervo Científico, 2021;23: 1-10

20. SANTOS VP, et al. Coexistência de psoríase e comorbidades relacionadas à síndrome metabólica. Rev. Soc. Bras. Clin. Med., 2016;14(2): 95-100

21. SCACCABAROZZI L, et al. Análise de custo por resposta dos medicamentos biológicos no tratamento da psoríase moderada a grave sob as perspectivas dos sistemas de saúde público e privado, no Brasil. Jornal Brasileiro de Economia da Saúde, 2016.

22. SILVA B, FARO A. Regulação emocional e sintomas depressivos em pacientes portadores de psíorise. Revista de Psicologia, 2019;28(2):1-10.

23. SILVEIRA ME. Perfil epidemiológico e qualidade de vida na psoríase. Revista Sociedade Brasileira de Clínica Médica, $2019 ; 15(4): 1-10$

24. SMEJKALOVÁ J, et al. Quality of life of patients with psoriasis. Cent Eur J Public Health , 2020, 28(3):219-225

25. SOCIEDADE BRASILEIRA DE SOCIEDADE BRASILEIRA DE DERMATOLOGIA (SBD). Consenso Brasileiro de Psoríase: algoritmo de tratamento da Sociedade Brasileira de Dermatologia. $3^{\underline{a}}$ ed, 2020. Disponível em: https://www.biosanas.com.br/uploads/outros/artigos_cientificos/152/770a01deea02365ae98071043abd3f12.pdf. Acesso em:21 de março de 2021. 